\title{
LA CITA Y LA REMINISCENCIA COMO FORMAS DE INTERTEXTUALIDAD EN EL RECURSO DEL METODO DE ALEJO CARPENTIER
}

María Salvadora Ortíz.

\begin{abstract}
In this article I explore two of the literary resources of baroque discourse: the quote and reminiscenses. I analyze these two resources in the novel El recurso del método, by Cuban author Alejo Carpantier. Theoretically I conceive of the text as a dialogic construction of the type proposed by Severo Sarduy and Mijael Backtine.
\end{abstract}

La cita y la reminiscencia son dos de los recursos propios del discurso Barroco. Severo Sarduy, quien toma los conceptos de Backtine, afirma que el texto Barroco es polifónico (1) debido al diálogo que en la obra establecen los diferentes textos, cuyos portadores son los actantes de que habla Greimas:

“...Espacio del dialoquismo, de la polifonía, de la camavalización, de la parodia, y la intertextualidad, lo barroco se presentaría pues, como una red de conexiones, de sucesivas filigranas cuya expresión gráfica no sería lineal, bidimensional, plana, sino en volumen, espacial y dinámica". (2)

La cita y la reminiscencia (3) son dos tipos de desviación que se dan en el discurso, las cuales se muestran al llevar a cabo un análisis suprasegmental de los enunciados en el texto de la novela. Hay dos tipos de enunciados novelescos la narración y las citas. La narración es un enunciado referencial, a partir de "un habla" asumida por el que escribe como actor-autor. Las citas son premisas textuales, a partir de "un habla" atribuida a otro:

"Le deuxième, type d'ecarts- la citation- vient d'un texte écrit. La langue latine et les "autres" (livres) (lus) pénetrant dans le texte du roman directement recopiés (citations) ou en tant que traces mnésiques (souvenirs). Il sont transportés intacts de leur propre espace dans l'espace du roman qui s'écrit recopiés entre guillemets ou plagiés" (4).

Estas citas y reminiscencias son utilizadas en todo El recurso del método. Para efectos de orden se clasifican las citas en dos grandes grupos: uno en que sólo se citan nombres, que se dividieron en cinco grupos por ser las citas más utilizadas por Carpentier; son nombres de lugares, nombres de medios de comunicación de masas, nombres de obras artísticas, nombres de personajes históricos y nombres de elementos relacionados con la música.

Un segundo grupo en que se citan textos directamente recopilados de un texto o del habla cotidiana. Estas citas son incorporadas intactas de su propio espacio al de la novela. Aquel espacio puede ser real o ficticio, aunque en su mayoría son tomados de la realidad. Hay citas de Proust que pertenecen al mundo de ficción.

\section{Citas de nombres de lugares:}

Tenemos por ejemplo la "Rue de Tilsitt", que corresponde a una calle de París cerca del Arco del Triunfo; aunque en los mapas sólo aparece con una "te". En esta calle "vivía" el Dictador, por lo que su nombre es citado varias veces (P. 108-P.297, etc.):

“...viniese a pasar los últimos días de su vida en esta siempre grata y consoladora mansión de la Rue de Tilsitt". (P.108).

En el siguiente cuadro se anotan algunos de los nombres de lugares que se citan en la novela. Se clasificaron en dos grupos, los que se ubican en América y los otros en Europa o E.E.U.U -ya que en todo el texto se da una oscilación del espacio "allá" y "aqui" que a veces es América y a veces Europa en relación con la ubicación del Dictador. 
Nombres de lugares

\begin{tabular}{|c|c|c|}
\hline AMERICA & EUROPA o E.E.U.U. & PAG. \\
\hline & Arco del Triunfo & 11,28 \\
\hline & Café Nachette & 21 \\
\hline \multirow[t]{2}{*}{ Cerro de las Campanas } & París & 25 \\
\hline & Passy, en Auteuil & 30 \\
\hline \multirow[t]{3}{*}{ Puerto Araguato } & & $27,31,12$ \\
\hline & Waldorf Astoria & 37 \\
\hline & Brentano's & 41 \\
\hline Cuba & $\theta$ & 42,44 \\
\hline El Surgidero de la Verónica & & 66 \\
\hline Santuario Nacional de la Divina Pastora & & 78 \\
\hline Nueva Córdoba & & 72 \\
\hline \multirow[t]{12}{*}{ Provincias Andinas } & & 12 \\
\hline & Torre Eiffel & 166 \\
\hline & Moulin Rouge & 166 \\
\hline & La Madeleine & 253 \\
\hline & Rue Tronchet & 253 \\
\hline & Chamonix & 267 \\
\hline & Rue Blomet & 290 \\
\hline & Gare Saint Lazare & 297 \\
\hline & Bois-Charbons & 303 \\
\hline & Quai Voltaire & 306 \\
\hline & Iglesia Saint Roch & 306 \\
\hline & Iglesia Saint Sulpice & 306 \\
\hline
\end{tabular}

Los medios masivos de comunicación que aparecen en El recurso del método corresponden al sistema capitalista, ya que obedecen, en gran parte, a las características del mercado de la información propio de los países literales

Nombres de algunos medios de comunicación de masas

\begin{tabular}{|c|c|c|}
\hline AMERICA & EUROPA ○ E.E.U.U. & PAG. \\
\hline & $\begin{array}{l}\text { Le Journal } \\
\text { L' Excelsior } \\
\text { L. Action Francaise } \\
\text { Le Petit Parisien } \\
\text { La Revue des Deux Mondes }\end{array}$ & $\begin{array}{l}12 \\
12 \\
12 \\
12 \\
28\end{array}$ \\
\hline $\begin{array}{l}\text { El Periódico Progreso } \\
\text { Barcelona }\end{array}$ & & $\begin{array}{l}31 \\
140\end{array}$ \\
\hline & L'Illustration & 142 \\
\hline Crítica & $\begin{array}{l}\text { Le Matin } \\
\text { Régiment (Revue) } \\
\text { Vanity Fair (Revue) } \\
\text { Saturday Evenning Post } \\
\text { New York Times }\end{array}$ & $\begin{array}{l}144 \\
145,146 \\
165 \\
199 \\
214 \\
217\end{array}$ \\
\hline $\begin{array}{l}\text { Buen Comentario } \\
\text { Liberación }\end{array}$ & & $\begin{array}{l}218,219 \\
224,251\end{array}$ \\
\hline & $\begin{array}{l}\text { L'Humanité } \\
\text { Le Figaro } \\
\text { Le Petit Parisien }\end{array}$ & $\begin{array}{l}307 \\
322 \\
322\end{array}$ \\
\hline El Mercurio & & 322 \\
\hline El Mundo & & 322 \\
\hline Ultimas Noticias & & 322 \\
\hline El Faro & & 322 \\
\hline El Centinela & & 322 \\
\hline El Machete (Revue) & & 322 \\
\hline
\end{tabular}


Dentro de estos medios se citan algunos que son de corte sensacionalista como Le Matin (P.94) que habla sobre el genocidio en Nueva Córdoba, hay otro de corte más objetivo y otros, como L'Humanité y Liberatión (P.224), que manejan un discurso objetivo.

Es importante anotar que dentro del texto el narrador define la información que cada uno de ellos da (5).

Veamos la descripción que de algunos periódicos se hace en el siguiente fragmento:

“Le Journal, ahora L'Excelsior, cuyas páginas, por sus muchas fotos, viene a ser un cinematógrafo de la actualidad. L'Action
Francaise, con las recetas gastronómicas de Pampille que mi hija señala cada día, con lápiz rojo, a la atención de nuestro excelente cocinero, y el inprecatorio editorial de León Daudet, cuyas geniales, apocalípticas injurias- expresión suprema de la libertad de prensa- promoverían duelos, secuestros, asesinatos y balaceras cotidianas en nuestros países" (P.12).

Nombre de obras artísticas (y de artistas) (6).

Alejo Carpentier poseía un vasto conocimiento de arte, incluso en Venezuela tuvo a su cargo la Cátedra de Historia del Arte. Esta erudición se manifiesta en sus textos. Estas citas de diferentes obras pictóricas, de escritores, novelas, etc. son puntos de referencia para ubicar el contexto cultural acorde al económico y dar al lector una imagen completa de aquel contexto.

Nombres de algunas obras artísticas y de artistas

\begin{tabular}{|c|c|c|}
\hline AMERICA & EUROPA o E.E.U.U. & PAG. \\
\hline & Escualtras de Rude & 12 \\
\hline & Marina de Elstir & 16 \\
\hline & Ninfa dormida de Gevex & 16 \\
\hline & El lobo de Gubbio de Luc Oliver Merson & 17 \\
\hline & Cena de Cardenales de Dumont & 17 \\
\hline Vista de Nueva Córdoba & & 17 \\
\hline Facundo (Sarmiento) & & 41 \\
\hline Zorrilla (España) & & 58 \\
\hline \multirow[t]{3}{*}{ Hokusai (Japón) } & & 97 \\
\hline & Sainte-Beuve & 97 \\
\hline & Verlaine & 97 \\
\hline \multirow[t]{5}{*}{ Rubén Darío-Amado Nervo } & & 96 \\
\hline & Oscar Wilde (Inglaterra) & 98 \\
\hline & Balzac & 155 \\
\hline & Rodin & 155 \\
\hline & Ajax, Edipo (Grece) & 160 \\
\hline Cabaña Tío Tom & & 214 \\
\hline \multirow{6}{*}{ David Griffth } & & 216 \\
\hline & Apollinaire & 217 \\
\hline & Los miserables & 231 \\
\hline & Gaston Leroux & 231 \\
\hline & Don Quijote (España) & 298 \\
\hline & Elstir & 306 \\
\hline
\end{tabular}


Para reafirmar lo antes dicho sobre la erudición de Carpentier creemos importante citar a Ramón García, quien ha realizado estudios al respecto:

"Las citas directas, las referencias de memoria y los juegos irónicos relacionados con las bellas artes son vastísimos y bien pueden justificar el aserto de que si Carpentier excede en conocimientos musicales y arquitectónicos, como se ha destacado hasta ahora, es, por otra parte, tal vez el escritor latinoamericano más apasionado por la pintura y la escultura, incluyendo a Cortázar" (7).

\section{Nombres de personajes relacionados con hechos históricos}

Estos personajes que se citan a través de todo el texto están relacionados sobre todo con hechos políticos. Los nombres de personajes históricos latinoamericanos son en su mayoría nombres de dictadores latinoamericanos y los europeos pertenecen en su gran mayoría a las clases dominantes.

\begin{tabular}{|c|c|c|}
\hline AMERICA & EUROPA o E.E.U.U & PAG. \\
\hline & Legión de Honor (groupe) & 14 \\
\hline & Rey Eduardo VII & 14 \\
\hline & Gran Boyardo & 15 \\
\hline Emperador de Brasil Pedro II & & 26 \\
\hline \multirow[t]{5}{*}{ Porfilio Díaz } & & 26 \\
\hline & Moltke (Allemagne) & 60 \\
\hline & Von Schlieffen (Allemangne) & 61 \\
\hline & Aníbal (Phénicien) & 61 \\
\hline & César, Ariovisto & 61 \\
\hline General Wood (Cuba) & & 72 \\
\hline Adelantado Sacho de Almeyda & & 72 \\
\hline \multirow[t]{3}{*}{ Gómez Carrillo } & & 96 \\
\hline & Bismark (Allemand) & 108 \\
\hline & Fichte (Allemand) & 117 \\
\hline El Dictador Rosas & & 129 \\
\hline Juan Vicente Gómez & & 113 \\
\hline \multirow[t]{4}{*}{ Estrada Cabrera } & & 155 \\
\hline & Paul Jamot & 156 \\
\hline & Aldo Nardini & 157 \\
\hline & Emesto Renán & 172 \\
\hline \multirow[t]{4}{*}{ Samuel Gompers } & & 178 \\
\hline & Metternich y Guizot (Autriche) & 190 \\
\hline & Robespierre & 200 \\
\hline & Bruant & 204 \\
\hline \multirow[t]{2}{*}{ Washington, Lincoln } & & 215 \\
\hline & Julio Veme & 214 \\
\hline Emiliano Zapata & & 232 \\
\hline \multirow[t]{3}{*}{ Mackandal } & & 232 \\
\hline & Calígula (Romain) & 243 \\
\hline & Enmanuel Kant (Allemand) & 286 \\
\hline Dictadores Latinoamericanos & & 300 \\
\hline Diego Rivera & & 322 \\
\hline Molla & & 326 \\
\hline \multirow{4}{*}{ Machado, Estreda Cabrera } & Nehrú (Inde) & 327 \\
\hline & Napoleón & 330 \\
\hline & Oswald Spengler (Allemand) & 331 \\
\hline & Aupick (Allemand) & 335 \\
\hline
\end{tabular}


Estas citas cumplen una función muy precisa dentro de El recurso del método: insertar los acontecimientos de la novela en la historia universal, como se verá con profundidad en la Segunda Parte concerniente a los Contextos.

Nombre de elementos relacionados con la música

Alejo Carpentier fue un gran musicólogo no sólo por el conocimiento que tenía de la música clásica sino también por el interés y profundidad en torno a la música latinoamericana. Tiene tres obras de ensayos dedicadas a la música: La música en Cuba, Letra y Solfa, y Ese músico que llevo dentro, obra póstuma. Es importante anotar que la música en la obra narrativa carpenteriana tiene gran relevancia en nivel semántico-estructural, como por ejemplo en la novela El acoso que está estructurada en forma de sonata: una primera parte, exposición, tres temas, diecisiete narraciones y conclusión. El tiempo de la novela son 46 minutos que corresponden al tiempo de duración de la Sinfonía Heroica de Beethoven; y así por ejemplo está también Concierto Barroco que desde su título mismo es una alusión a la música.
PIEZAS

MUSICALES

El faisán

MUSICOS

Nombres de algunos elementos musicales

\begin{tabular}{|c|c|c|c|}
\hline & AMERICA & EUROPA o E.E.U.U. & PAG. \\
\hline \multirow{15}{*}{$\begin{array}{l}\text { PIEZAS } \\
\text { MUSICALES }\end{array}$} & Vals de Juventino Rosas o de Lerdo de Tejada & & 19,163 \\
\hline & & Cosí fan Tutte & 91 \\
\hline & & Marcha de Tannhausser & 112 \\
\hline & & Claro de luna & 145 \\
\hline & & Vals de Waldteufel & 160 \\
\hline & & Marcha de Sousa & 165 \\
\hline & & Ballet Sansón y Dalila & 183 \\
\hline & & Pasodobles Toreros & 183 \\
\hline & & Barbero de Sevilla & 197 \\
\hline & & Rigoletto & 199 \\
\hline & & La Sonámbula & 199 \\
\hline & & La Favorita & 199 \\
\hline & & Martha de Flotow & 199 \\
\hline & & Hamlet & 199 \\
\hline & & La Tosca & 199 \\
\hline \multirow{19}{*}{ MUSICOS } & El faisán & & 316 \\
\hline & Alma Campera & & 316 \\
\hline & El Tamborito & & 316 \\
\hline & Flores Negras & & 316 \\
\hline & Las perlas de tu boca & & 316 \\
\hline & La milonguita & & 19,163 \\
\hline & Juventino Rosas & & 19 \\
\hline & Lerdo de Tejada & & 19 \\
\hline & Instrumentos musicales latinoamericanos & & 66 \\
\hline & & Toscanini & 197 \\
\hline & & Umberto Giordano & 199 \\
\hline & & Tito Schipa & 229 \\
\hline & & Beethoven & 169 \\
\hline & & Mozart & 91 \\
\hline & & Diaghlen & 307 \\
\hline & & Zez Confrey & 307 \\
\hline & & Haych & 163 \\
\hline & & Mendessohn & 163 \\
\hline & & Raff & 163 \\
\hline
\end{tabular}


Salvador Bueno, crítico cubano, dice al respecto:

"El gran novelista cubano aprovecha para su quehacer literario las fórmulas y la temática de dos disciplinas: la música y la arquitectura. He dejado aclarado que: "El problema de la forma, en música, me ha preocupado mucho, siempre. Y he tratado de hacer transformaciones de conceptos formales musicales a conceptos formales literarios" (8).

En el cuadro anterior se anotan elementos relacionados con la música, sin embargo estos nombres no arrojan la perspectiva que de ella se tiene en el texto de la novela. En este cuadro no aparecen las citas de estrofas de música folklórica y protesta, que en América Latina son muy significativas y que, en conjunto, se analizarán en lo referente al Contexto Artístico.

Estas citas de elementos musicales ayudan a ubicar la producción musical latinoamericana de la época (1913-1927) dentro del contexto musical europeo, y ver la relación de influencia que se gestó en este período de Europa con respecto a América.

En El recurso del método se dan dos tipos de citas: las de nombres de lugares, de medios de comunicación de masas, de obras artísticas y de artistas, etc.; y las citas extensas, que son frases o fragmentos recopilados en forma textual en la novela. Este segundo tipo de citas se agrupan para su análisis, al igual que las primeras, de acuerdo con dos de los argumentos que contribuyen a formar el anillo temático de la novela: América-Europa, anillo oposicional que enmarca el enunciado novelesco.

Son varias las citas textuales que provienen de textos escritos, tenemos por ejemplo una cita de un libro de Historia en que se relata algunas de las costumbres de la Galia, entre las que estaba la de tener partidos políticos, hecho al cual el Dictador atribuye su derrota:

\footnotetext{
“Y, en espera del sueño, corrió las páginas, hasta dar con un Sexto Libro, cuya lectura le había quedado interrumpida semanas antes. Capítulo XI: "Ya que alcanzamos esta parte del relato nos parece oportuno extendernos sobre las costumbres de la Galia y de la Germania y sobre las diferencias que distinguen dichas naciones. En Galia, no solamente en cada estado, sino también en cada pequeña comarca, y hasta en el seno de cada familia hay partidos"... Hay partidos. -"Por eso es que los jodieron..." (Europeo)(P.55).
}

También tenemos citas de textos clásicos, "humorísticamente citados"; en este caso una del Dictador para dárselas de culto:

"soplad, vientos, y romped vuestras mejillas. Y vosotros, relámpagos activos como la idea, anunciadores del rayo que raya las encimas, venid a enrojecer mi nívea cabeza..." (P.58) (Europeo).

Y luego le responde con otra cita el Doctor Peralta, dicho poema era a menudo recitado en el Teatro Nacional:

“ ¿Qué tormente nos amaga! ¿Qué noche, válgame el cielo! Ciego es el terrible acento y el fulgor que centelles cuando sopla airado el viento y el cénit relampaguea?..." (Europeo) (P.59).

Los anuncios son otro tipo de texto que se recopia, véanse algunos (9).

\section{América}

“...el Compuesto Vegetal de Lydia Pinkham -retratada con gola y camafeos- soberano en asuntos de trastornos menstruales". (P.46).

Otro anuncio propagandístico es el de las sábanas benditas para recién casados que se hacen en el barrio Marais, en París, pero que también en Amé-rica se usan:

“...el negocio de las "Sábanas benditas para Recién Casados" (Draps-bénis pour jeunes mariés)” (P.184).

Tenemos también anuncios en que se incorpora en forma textual la voz del pueblo; pero en éste es lo oral, noloescrito, lo que se incorpora, esto es cuando el país está en crisis y el Edificio de la Bolsa de Valores se convierte en Mercado.

"-A usté, para el azúcar en la sangre, cocimiento de albahaca morada; a usté, para el asma, cigarros de campana doble; a usté, para el humor que le sale del miembro, agua de coco con ginebra holandesa; a usté, comadre, para la luna retrasada, un té de cundiamor, con dos hojas de almácigo, ahí, dispensando el modo de señalar donde se le abre la tatagua..." (América)(P.246).

Otro anuncio que proviene de un espacio escrito es cuando el pueblo escribe en las paredes, muros, etc. "¿Qué se vaya! ¡Qué se vaya! (América). (P.260).

Otros textos que se incorporan a El recurso del método son los de Carlos Marx; unos tomados de $E l$ Capital como el de las páginas 188-189, otras del Manifiesto Comunista en las páginas: 224 y 256; otros de la Contribución a la crítica de la Economía Política en la página 230. Y el siguiente de Salario, precio y provecho que dice así:

"No creo que esto sea capaz de pervertir a nuestros conductores de tranvías... 
Marx: Salario, precio y provecho. Y leyó el Presidente: La determinación de los valores de las mercancías mediante cantidades relativas de trabajo que le son incorporados es algo totalmente distinto del método tautológico de la determinación de los valores de las mercancías por el valor del trabajo o por los salarios". (Europa)(P.188).

Otros textos, que se incorporan literalmente, son textos de tema bélico como los de Moltke y Schlieffen, alemanes, sobre las reglas de empleo de las armas (cfr. P.60). También de Foch (francés) siempre sobre el mismo tema. P.66.

Sobre la guerra y sus estrategias, tenemos textos de Lassau y Scharhorst (P.75). También de estrategias bélicas, en la página 248, de Maurice Barrés, conservador radical, que dice así:

\begin{abstract}
"Reina el buen humor en las trincheras. Claro que allí, en las noches lluviosas, no se está como en un restaurante de lujo... Pero conozco un lugar donde, en un laberinto de ocho kilómetros de trincheras muy cuidadas, los caminos se designan por los nombres de Champs-Elysées o la Rue Monsieur-le-Prince. Sé de un abrigo subterráneo donde un oficial posee una butaca de terciopelo carmesí, una mesa con ramos de rosas y platos de viejas porcelanas de Estrasburgo. Las trincheras se adornan con muebles hallados en las ruinas de los pueblos bombardeados reina la alegría en las trincheras" (Sic)" (Europa)(P.166).
\end{abstract}

Otros textos que se incorporan son de escritores como Rubén Darío (P.97), Oscar Wilde (P.98) y Baudelaire (P.289).

De la voz del pueblo se incorporan al texto varias estrofas de música popular latinoamericana, que tienen como objetivo resaltar la identidad musical latinoamericana.

En $E l$ recurso del método, se citan canciones populares en siete diferentes situaciones. Primero cuando en el levantamiento de Ataúlfo Galván el Primer Magistrado y los soldados se dirigen en tren al interior del país, para vencer a los sublevadores y en el viaje los soldados iban cantando canciones mejicanas; las cuales son muy conocidas por el pueblo; sobre todo en la zona caribeña. Las estrofas que se citan son:

\footnotetext{
"Adiós, adiós

Lucero de mi vida,

Dijo un soldado

al pie de una ventana" (P.54).
}

\footnotetext{
"La noche que la mataron

Rosita estaba de suerte

De seis tiros que le dieron,

No más uno era de muerte..." (P.55)
}

\section{Y esta otra dentro de un contexto geográfico muy} caribeño:

"Era media noche casi cuando salió el último tren, entre silbidos y escapes de válvulas. Sobre el techo de los vagones y tercerolas cantaban hombres de ruanas y mujeres de rebozo, concertados en himnos y canciones, mientras las botellas de ron blanco corrían, a la luz de las linternas y faroles, de los carbones de ténder a los ojos encendidos del furgón de cola: Qué si Adelita se juese con otro, la seguiría por tierra y por mar; si por mar en un buque de guerra; si por tierra, en un tren militar... Y, detrás, la noche de los sapos en las marismas negras del Surgidero..." (P.73).

La segunda situación es el levantamiento de Walter Hoffman en que se oye también la voz del pueblo cuando el Dictador está en su casa en América Latina. La canción es entonada en Semana Santa mientras se hacía la representación del Viernes Santo. Mientras el actor, que hacía de Cristo, subía por la Calle de la Amargura el pueblo cantaba:

"Y había seguido su camino, cuesta arriba, por la Calle de las Amarguras mientras la multitud coreando una vieja melodía de no se sabía donde, cantaba, con lentas inflexiones de canto llano:

"Y si he de morir mañana que me maten de una vez" ". (P.131).

Otra situación es a raíz del barco alemán Lubeck en que toman presos a sus tripulantes y los trasladan a una finca y éstos rápidamente se organizan, forman un conjunto musical, construyen sus casas, etc. Lo que es importante resaltar acá es que ellos no toman nada de la cultura latinoamericana, siguen con sus costumbres y para mostrar esto se cita una de las canciones que ellos cantaban:

"Y a menudo, muy bien acompañada por el conjunto, cantaba el sobrecargo del Lubeck, con su linda voz de tenor:

Winterstruirmen wichen

dem Wonnemond

in milden lichte

leuchtet der Lenz" (P.164).

Cuando concluye la Guerra (1919) en el país del Dictador, el pueblo se lanza a la calle a festejar. El pueblo canta diversas canciones: unas de confluencia árabe, debido al substrato cultural colonial español , como esta:

"El Kaiser corcovea y Yoffre lo menca" (P.192).

Otras que muestran la fucrte influencia cristiana en el pueblo como:

"Santa María

Líbranos de todo mal 
ampáranos, Señora

De este tremendo animal" (P.192)

Y esta:

"La Virgen cogió un machete para poderlo matar y el Demonio en "cuatro patas se metió al matorral" (P.192).

Es importante destacar que, mientras en el texto se incorporan canciones que son signos de la identidad latinoamericana y es el pueblo quien las canta, el Dictador toma distancia y escucha música francesa:

"Lorsque la nuit tombe sur Paris. La belle église de Notre Daame samble, monter au paradis, pour lui conter son etat d'aaame" (P.193).

Con esta cita se manifiesta cual es en parte la identidad cultural del Dictador, y cuál es su preferencia en lo concerniente a la música.

Después de la guerra también se van a dar una serie de cambios en el nivel cultural. Los Estados Unidos inician sus relaciones comerciales con los países latinoamericanos (10) y éstas implican una serie de cambios en las costumbres de estos últimos. Se rompe esa exclusividad de metrópoli y se ve como hasta las canciones de Navidad se cambian por las norteamericanas como se muestra en esta cita:

"Las Navidades tradicionales, las de la Colonia, las de ayer, las de siempre, fueron desalojadas en un día por las Navidades Nórdicas. Aquel año no salieron a las calles las bullangueras parrandas de pandero y villancico, para visitar al vecindario al compás de un "Tún-tun... ¿Quién es...? Gente de paz", cuyos cantores iban culebreando por las calles... las canturías de otros tiempos fueron sustituidas, en las casas decentes, por cajitas de música que tocaban las melodías de Silent night, holy night o Twinkle, Twinkle, little star..." (P.221).

Esta influencia de la cultura nórdica no sólo se da en América Latina, también en Europa, y se muestra en el momento que el Dictador es derrocado y regresa a París para quedarse ahí. La noche que llega su hija Ofelia viene de una fiesta, y la continúan en la casa del Presidente, ponen un poco de música y se escucha tanto la francesa como la norteamericana. Véase las canciones que se citan:

"Yes, we have no bananas..." (P.302).

La anterior aparece varias veces. La siguiente es una de las canciones de moda en esa época:
"Je cherche aprés Titine, Titine, joh! ma Titine..." (P.302).

Las últimas dos estrofas de canciones son las que se citan cuando Ofelia se acuerda de su niñez, en la cena que preparó Elmirita y son las mismas que se citan cuando el pueblo celebra el fin de la Primera Guerra Mundial (11).

Este análisis de las citas, como enunciados mediatizadores y característicos del discurso Barroco, son desviaciones que en $E l$ recurso del método cumplen con la función que plantea Alejo Carpentier en sus ensayos: "Y me pregunto ahora si la mano del escritor puede tener una misión más alta que la de definir, fijar, criticar, mostrar el mundo en que le ha tocado en suerte vivir. Naturalmente, para ello hay que entender el lenguaje de ese mundo" (12).

Estas citas a la vez que mediatizan la comunicación ayudan a que se comprenda mejor la cultura latinoamericana dentro de un contexto universal.

Las citas de nombres son en su mayoría nombres que pertenecen a elementos europeos y las citas extensas corresponden la mitad, aproximadamente, a América y la otra a Europa, lo que contribuye a insertar una serie de elementos culturales latinoamericanos, como las estrofas de canciones.

\section{NOTAS}

(1) El término polifónico surge con el estudio que hace Backtine sobre la obra de Dostoievski. Este aspecto es fundamental en la poética de su obra.

La polifonía del texto romanesco no se puede resumir en unas pocas líneas. Lo plantea Backtine y Kristeva lo retoma en El texto de la novela (P. 94-97).

(2) Sarduy, Severo. "El Barroco y el Neobarroco: América Latina en su literatura. Editorial Siglo XXI, México, 2 da. Edición, 1974. P.175.

(3) Kristeva establece dos tipos de análisis para extraer el ideologema del signo: suprasegmental y el intertextual.

(4) Julia Kristeva. Semiotique (Recherches pour une sémanalyse). (Extraits) Edition du Seuil, Paris, 1969. P.74.

(5) Cfr. las diferentes páginas en que aparecen los nombres de los medios de comunicación masiva.

(6) Ramón García Castro hace una descripción de cada una de las obras pictóricas que aparecen en El recurso del método, Concierto Barroco, y Los convidados de plata. 
(7) Ramón García Castro. Notas sobre la pintura en tres obras de Alejo Carpentier: Los convidados de plata, Concierto Barroco y El recurso del método. Revista Iberoamericana, Vol.XLVI, enero-junio 1980, Números 110-111. P.67.

(8) Salvador Bueno. De Merlin a Carpentier. Unión de Escritores y Artistas de Cuba, Cuba, 1977. P.231.

(9) Las citas textuales en el texto de la novela aparecen en letras bastardilla nosotros las subrayamos. También tenemos una cita de la Biblia P.277, de la Constitución del país del Dictador, P.218, de epitafios P.128.

(10) Cfr. de Eduardo Galeno: Las venas abiertas de América Latina.

(11) Cfr. P.315 de El recurso del método.

(12) Alejo Carpentier: La novela latinoamericana en vísperas de un nuevo siglo y otros ensayos. P.45.

\section{BIBLIOGRAFIA}

Sobre la obra de Alejo Carpentier:

Arias, S. (Comp). "Recopilación de textos sobre Alejo Carpentier", Casa de las Américas. Cuba: 1975 , p.585.

Bueno, S. "Carpentier en la maestría de sus novelas y relatos", Revista de la unión de escritores y artistas de Cuba (1). Année 13, mars 1974, pp. 101- 121.

Gribanov, A. "El nombre como factor constructivo en las novelas de Alejo Carpentier”, América Latina 2 (26). Moscou: Editorial Progreso, 1980.

Leante, C. "Confesiones sencillas de un escritor
Barroco", Revista mensual Cuba 2 (24). La Havane: Abril 1964.

Mujica, H. “Alejo Carpentier y la responsabilidad del escritor”, Casa de las Americas (129) La Havane: nov.-déc. 1981, pp.57-67.

Muller-Bergh, K. (ed.) Alejo Carpentier: estudio biográfico-crítico. New York: Las Américas pub. Co. 1972.

Muñoz, M. "El eterno retorno y los símbolos de creación y destrucción en "Los pasos perdidos" de Alejo Carpentier", Universidad de Antioquía 68 (188), julio-sept. 1973, pp. 273-276.

Pickenhayn, J.O. Para leer a Alejo Carpentier. Argentina: Editorial Plus Ultra, 1978, p.174.

Rodríguez, I. "Historia y alegoría en Alejo Carpentier", Hispanoamérica (17) Argentina: pp.23-43.

Sud, (Série Colloques) Alejo Carpentier et son oeuvre 1. Marseille: Centre National des Léttres, 1982, pp. 49-61.

Vásquez, C. "Alejo Carpentier, historien de son époque", Sud (Série Colloques). Alejo Carpentier et son oeuvre. Centre National des Léttres (12), 1982, pp. 62-76.

Sobre El recurso del método.

Labastida, J. “Alejo Carpentier: realidad y conocimiento estético sobre 'El recurso del método' ", Casa de las Américas (87). La Havane: nov-dec. 1974. 
